\title{
MANEJO DE LA ATONIA UTERINA EN UN HOSPITAL PROVISIONAL POST TERREMOTO. ICA, PERÚ.
}

\author{
Jorge Ybaseta-Medina ${ }^{1, b} \quad$ Karoly Rios-Lopez ${ }^{2, a}$, Heberth Ajalcriña ${ }^{2, a}$, Marjorie Ybaseta-Soto ${ }^{3, b}$ \\ Garamende Juan $^{3, b}$, Daniel Medina-Atoche ${ }^{2, b}$, Katherine Garcia-Cornejo ${ }^{2 b}$, Maritza Lizaraso-Chacon ${ }^{1}$
}

1. Gineco-obstetra del Hospital Santa María del Socorro de Ica.

2. Internos de Medicina del Hospital Santa María del Socorro de Ica.

3. Medico Cirujano

a. Universidad San Juan Bautista. Lima, Perú.

b. Universidad Nacional San Luis Gonzaga. Ica, Perú.

\section{RESUMEN}

Se reporta el caso de una mujer de 20 años de edad, sin antecedentes de importancia. Fue admitida en el hospital, en fase activa de trabajo de parto con una gestación de 36 semanas; luego del parto vaginal, presentó hemorragia posparto, compromiso del estado general. A pesar de las medidas físicas y farmacológicas protocolizadas, continuó con hemorragia profusa presentando shock hipovolémico. Se administró rápidamente cristaloides a doble vía a goteo rápido continuándose con coloides, con adaptación a las condiciones del hospital provisional, sin demora se realizó histerectomía evitándose morbilidad grave o mortalidad materna.

Palabras Clave: Hemorragia Posparto, Histerectomía, Shock Hipovolémico, Mortalidad Materna. (fuente DeCS BIREME)

\section{MANAGING OF THE UTERINE ATONY IN A PROVISIONAL HOSPITAL POST EARTHQUAKE. ICA, PERU}

\begin{abstract}
It is report the case of a 20 -year-old woman, without precedents to importance. She was admitted into the hospital in labor active phase with a gestation of 36 weeks, after the vaginal childbirth she had postpartum hemorrhage, general state of health. Despite physical measures and pharmacological, she continues with bleeding profusely and presenting hypovolemic shock. It was administered rapidly crystalloids to double intravenously to rapid dripping being continued by colloids with adjustment to the conditions of the provisional hospital, without delay it was realized hysterectomy avoiding severe morbidity or maternal mortality.
\end{abstract}

Key words: Postpartum Hemorrhage, Hysterectomy, Shock, Maternal Mortality. (source MeSH NLM).

\section{INTRODUCCIÓN}

El 15 de agosto de 2007 ocurrió un sismo de magnitud VIII en la escala de Mercalli Modificada en la Región Ica, el más intenso ocurrido en el Perú en los últimos cien años, obligando a que la población de la provincia de lca se atienda en hospitales de campaña mientras construían hospitales nuevos. La Región Ica abarca una superficie de $21,328 \mathrm{~km}^{2}$ y una población de 711932 habitantes ${ }^{(1)}$. Sufrir el terremoto constituyo una experiencia similar a la vivida en la República Islámica de Pakistán donde el 8 de octubre del 2005 ocurrió un terremoto de gran intensidad que afectó $28000 \mathrm{~km}^{2}$, allí la ayuda de Cuba no tardó en llegar, desplegando un hospital de campaña en la ciudad de Muzaffarabad, con 880000 habitantes, qué tenia una infraestructura sanitaria pésima dada por el $66,1 \%$ de sus hospitales inhabilitados ${ }^{(2)}$. Las operaciones cesáreas fueron la principal cirugía de urgencia y en las ginecológicas sólo una cirugía por atonía uterina en 6 meses de instalados ${ }^{(2)}$. Tuvieron datos parecidos a los de uno de los hospitales provisionales de Ica donde, en el tiempo de 8 meses, hubo dos histerectomías sin complicaciones una por atonía uterina y otra por placenta acreta. Estos datos fueron también similares a los reportados por otras brigadas médicas cubanas que trabajaron en Honduras, entre noviembre de 1998 hasta septiembre de 1999 en ocasión del azote a ese país por el huracán Mitch ${ }^{(3)}$.

Estas se constituyen en experiencias adquiridas, a través de los años, en múltiples situaciones de emergencias y desastres vividas en diferentes países, en donde se ha podido demostrar las graves debilidades en cuanto ha: la preparación, prevención, mitigación, respuesta, reconstrucción y rehabilitación para casos de desastres por parte de muchos gobiernos ${ }^{(4)}$.

La atonía uterina sigue siendo una de las causas más frecuentes de shock hipovolémico y está íntimamente ligado a la morbimortalidad materna, actualmente la mortalidad materna en el Perú se estima en 160 por 100,000 nacidos vivos siendo la hemorragia por atonía la primera causa de muerte materna ${ }^{(5,6,7,8,9,10)}$. Por tanto la atonía uterina es un tema de gran importancia para disminuir la mortalidad materna en el Perú. En lca la tasa de mortalidad materna de 37,17 por 100,000 nacidos vivos, todas probableme 
evitables ${ }^{(11)}$. En caso de desastre existe una situación de calamidad, miseria y carencia de la infraestructura sanitaria (2). De ahí que el objetivo de este reporte es determinar el comportamiento del manejo de la atonía uterina en un hospital de campaña post desastre.

\section{CASO CLINICO}

Paciente de 20 años de edad, con G1p1, controlada en un puesto de salud, se interna en un hospital provisional post terremoto, con diagnóstico trabajo de parto, gestación de 36 semanas y periodo intergenésico corto, refiriendo dolor abdominal tipo contracción uterina. La exploración física reveló: Presión Arterial 100/60 mmHg, Frecuencia Cardiaca 72x', Respiración 20x', afebril. A la evaluación por órganos aparatos y no se evidenció alteraciones.

Examen Obstétrico: Abdomen con útero grávido a $3 \mathrm{~cm}$ por debajo del apéndice xifoides, con feto en posición longitudinal cefálico derecho, altura uterina de $34 \mathrm{~cm}$, Frecuencia Cardiaca Fetal 140x' rítmicos, Peso ponderado fetal $3410 \mathrm{~kg}$. dinámica uterina $3 / 10^{\prime}+++$; tacto vaginal dilatación 7cm., Incorporación $80 \%$ APF -3, membranas amnióticas integras, pelvis ginecoide. El hematocrito preparto fue de $32 \%$ y $\mathrm{Hb}$ de $10.6 \mathrm{gr} / \mathrm{dl}$. Tuvo parto vaginal obteniéndose un recién nacido de 3620 gr, Apgar 8-9. Se realizó alumbramiento dirigido se advierte membranas disociadas y hemorragia profusa 600cc. Se apoyó hemodinámicamente con 2 litros de cristaloides, oxitócicos y masaje uterino. Posteriormente se procedió a legrado y escobillonaje de la cavidad uterina en sala de parto extrayéndose poca cantidad de membranas ovulares y se sutura un desgarro vulvoperineal de II grado. Sangrado durante el procedimiento $200 \mathrm{cc}$, quedando con presión arterial de $90 / 60 \mathrm{mmHg}$ y pulso de 110 por minuto.

La paciente 30 minutos después se muestra con impresionante palidez, sensación de vértigo, útero hipotónico al examen bimanual se extraen abundantes coágulos sanguíneos, pasa a sala de operaciones con doble vía administrándose cristaloides y coloides a goteo rápido en una relación 2:1; también se administró, oxitocina, misoprostol 800 mcgs rectal y masaje uterino. Se realiza nueva revisión de vagina, suturando desgarro de cuello uterino, sin embargo continúo el sangrado profuso proveniente de cavidad uterina, se colocó sonda Foley. La evolución negativa de las funciones vitales frustra el taponamiento uterino con un balón que se construyó insertando una sonda Nelaton número 18 en un preservativo para que haga la misma función de un balón bakrí. Precavidamente el único ginecólogo de guardia que estaba trabajando con el interno de medicina, previa llamada ya había enviado la ambulancia para traer a otro ginecólogo que no estaba programado; se informa a los familiares sobre eventuales complicaciones, estos otorgan el consentimiento para la histerectomía total en virtud del shock hipovolémico $y$, de esta manera evitar la muerte materna. Durante la intervención quirúrgica, en la medida de lo posible, se hace presión manual en dirección a la arteria aorta y las uterinas. Las funciones vitales se estabilizan continuando así en el post-operatorio. Se indican analgésicos, antibióticos y no contándose con paquete globular se indican dos unidades de sangre fresca. Teniendo una Hemoglobina de 5.3 grs \% posterior a la primera transfusión sanguínea. Se otorga el alta al cuarto día del puerperio con una Hemoglobina de $7 \mathrm{gr} \%$, seis meses después la paciente hace su vida sexual normal, el hijo recibe lactancia materna sin ninguna dificultad.

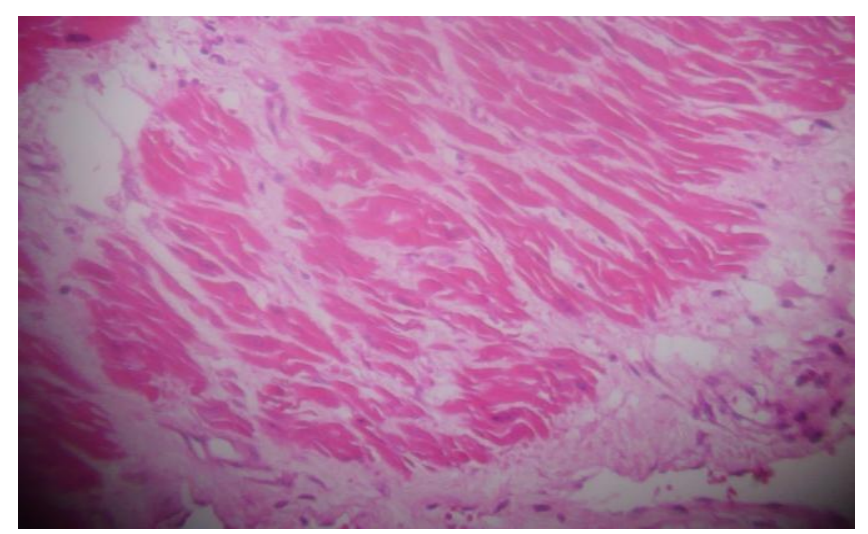

Figura 1. MICROSCOPIA 40X

Se observa pared uterina constituida por fibras musculares lisas algunas de ellas con necrosis, y vasos sanguíneos delgados dilatados.

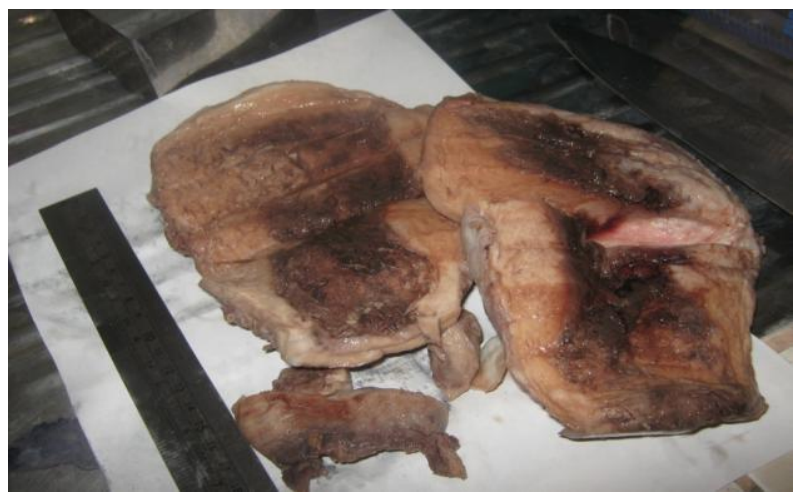

Figura 2. MACROSCOPIA

Útero, se observa endometrio con zonas de hemorragia y necrosis, no se observa tejido placentario.

\section{DISCUSIÓN}

La atonía uterina constituye la causa mas frecuente de hemorragia siendo responsable desde el $29,5 \%$ hasta del $80 \%$ de las histerectomías post parto ${ }^{(11,12)}$ congruente con lo reportado en los hospitales de campaña en lca y Muzaffarabad, Pakistán ${ }^{(2)}$.

Las causas que generan la atonía uterina son identificables siendo su diagnóstico clínico lo que permite que el manejo médico sea oportuno y obligatoriamente eficaz ${ }^{(12)}$. El manejo para conservar el útero en estas pacientes hasta ahora varía ampliamente y consiste en manejo médico, 
de retractores uterinos, ligadura de arterias uterinas y/o hipogástricas, suturas al lecho placentario, embolización de arterias uterinas y uso de métodos mecánicos de compresión como packing de compresas y dispositivos intrauterinos; en caso de fallo de estas medidas conservadoras sería peligroso multiplicar técnicas y debe realizarse histerectomía postparto conservando los anexos ${ }^{(13,14)}$; también, cuando no se tienen los recursos para un manejo conservador y existe hemorragia profusa o en situaciones de peligro para la vida debe realizarse la histerectomía antes que las condiciones de la paciente sean extremas a fin de evitar morbilidad grave 0 la muerte materna $^{(15,16)}$.

En conclusión el resultado del manejo médico y quirúrgico en un hospital provisional post desastre es comparable con el que se obtiene en condiciones normales de trabajo.

\section{Agradecimiento:}

A Lidia Quispe Galindo Médico Anátomopatologa por su aporte en lo que concierne a su especialidad.

Correspondencia:

Daniel Medina Atoche

Correo electrónico: rev.med.panacea@unica.edu.pe

Teléfono: 956-606880

\section{REFERENCIAS BIBLIOGRÁFICAS:}

1. Perú, Instituto Nacional de Estadística e Informática. Compendio Estadístico Departamental 2008-2009. Disponible en: http://www.inei.gob.pe/biblioineipub/bancopub/Est/Lib0 839/libro.pdf

2. Negrín R, Martín J, G García, Castillo L. Cirugía de urgencia en un hospital cubano integral de campaña en una zona de desastre. Rev Cub Med Int Emerg 2007;6(4):881-887.

3. Rosales González SO, Lombardo Vaillant A, de la Horma PM. Trabajo médico quirúrgico en un país de Centro América en situación de desastre. Rev. Cubana Med. Milit 2003; 32(4).

4. Press F, Hamilton R. M. Mitigation emerges os major strategy for reducing losses caused by natural disasters. Board of natural disasters. Science 1999; 284(5422): 1943-7.

5. Organización Panamericana de la Salud. Más allá de la supervivencia: Prácticas integrales durante la atención de parto, beneficiosas para la nutrición y la salud de madres y niños. Washington, D.C.: OPS; 2007.

6. Mc Cormickml. Preventing pospartum hemorrhage in low_resourse settings.Inter Ginecol Obstet. 2002 77:267-275
7. Schwarcz R, Fescina R, Duverges C. El parto patológico. Obstetricia. 6a ed. Bs As: El Ateneo, 2008: 629-644.

8. Martinez-Galiano JM. Prevención de la hemorragia posparto con el manejo activo del alumbramiento. Matronas prof 2009; 10 (Supl 4): 20-26.

9. Rivero M, Avanza MJ, Alegre MC, Feu M, Valsecia M, Schaab A, y col. Hemorragia postparto: incidencia, factores de riesgo y tratamiento. Comunicaciones científicas y tecnológicas UNNE [en línea] 2005 [fecha de acceso 02 marzo 2011]; 3. Disponible en: http://www.unne.edu.ar/Web/cyt/com2005/3-Medicina/M-

10. Karisson H, Perez C. Hemorragia postparto.. An Sist Sanit Navar 2009; 32 (Supl 1): 159-167.

11. Ybaseta-Medina J. Factores de riesgo asociados a la mortalidad materna en la Región de Ica, Perú. 20012005. Rev. méd. panacea 2011, 1:9-13.

12. Tamizian $\mathbf{O}$, Arulkumaran $\mathbf{S}$. The surgical management of post-partum haemorrhage. Best Pract Res Clin Obstet Gynaecol 2002;16(1):81-98.

13. Bakri YN, Mari A, Abdul Jabbar F. Tamponade-balloon for obstetrical bleeding. Int J Obstet 2001; 74(2): 139-42.

14. B-Lynch C, Coker A, Lawal AH, Abu J, Cowen MJ. The B-Lynch surgical technique for the control of massive haemorrhage: an alternative to hysterectomy? Five reported. Br J Obstet Gynaecol 1997; 104(3); 3725.

15. Mitelman G, La Rosa G, Martínez F, Cadima R. Alternativa quirúrgica en la hemorragia grave del puerperio. Rev Chil Obstet Ginecol 2004; 69(4): 316-18.

16. Ramón M. Miralles Atonía uterina como causa más frecuente de histerectomías postparto Ginecología y Obstetricia Clínica 2006;7(1):10-15

Recibido: 07/08/2011

Aceptado para publicación: 24/02/2012 